\title{
Fish Myogenic Regulatory Protein LUC7L: Characterization and Expression Analysis in Korean Rose Bitterling (Rhodeus uyekii)
}

\author{
Ju Lan Kim, ${ }^{\dagger}$ Hee Jeong Kong, Hyung Soo Kim, Woo-Jin Kim, Dong-Gyun Kim, Bo-Hye Nam, \\ Young-Ok Kim and Cheul Min An \\ Biotechnology Research Division, National Fisheries Research and Development Institute, Busan 619-705, Korea
}

\begin{abstract}
Serine-arginine-rich nuclear protein LUC7L plays an important role in the regulation of myogenesis in mice. In the present study, we isolated and characterized the Korean rose bitterling Rhodeus uyekii Luc71 cDNA, designated RuLuc71. The RuLuc71 cDNA is 1,688 bp long and encodes a 364-amino-acid polypeptide containing serine/arginine-rich region at the C-terminus. The deduced RuLuc71 protein has high amino acid identity (71-97\%) with those of other species including human. Phylogenetic analysis revealed that RuLUC7L clustered with fish LUC7L proteins. The expression of RuLuc71 mRNA was high in the brain, kidney, and stomach of Korean rose bitterling. Expression of the RuLuc71 mRNA was detected from 1 day post-fertilization (dpf) and moderately increased until $21 \mathrm{dpf}$ during the early development. Further investigations are required to elucidate the functional role of RuLUC7L in myogenesis in R. uyekii.
\end{abstract}

Key words : Development, Korean rose bitterling, Luc71, Myogenesis, Rhodeus uyekii

\section{INTRODUCTION}

Myogenesis (or muscle development) is composed of the specification processes of the individual myogenic progenitor cells according to myogenic lineage, proliferation and migration, differentiation, and fusion. The various myogenic regulatory factors (MRFs) are involved in the commitment of mesodermal cells to a muscle lineage (i.e., MyoD, Myf5) and in the initiation and maintenance of the terminal differentiation program (i.e., Myogenin, Mrf4) (Arnold \& Braun, 1996). MRFs are highly conserved in teleost and mammals and whose mutation will result in muscular defects (Hasty et al., 1993; Megeney et al., 1996; Rescan, 2001; Kassar-Duchossoy et al., 2004). Although there are several common features in muscle development between teleost and mammals, myogenesis in teleost has some unique features compared to mammals, which include the early stage of muscle commitment, presence of adaxial cells, different proportions of slow and fast fibers, and muscle growth throughout much of ontogeny (Rossi \& Messina, 2014). Whereas muscle development in mammals is conventionally divided into pre-natal and post-natal, the main phases of myogenesis in teleost consist of the embryonic-larval-juvenile and adult stages (Johnston et al., 1998; Rossi \& Messina, 2014).

The LUC7-like gene (Luc7l) encodes a putative RNAbinding protein similar to the yeast Luc7p subunit of the U1 snRNP splicing complex, which has a role in $5^{\prime}$ splice

\footnotetext{
Manuscript received 10 November 2014, Received in revised form 20 November 2014, Accepted 24 November 2014

${ }^{\dagger}$ Corresponding Author: Hee Jeong Kong, Biotechnology Research Division, National Fisheries Research and Development Institute (NFRDI), 216 Haean-ro, Gijang-up, Gijang-gun, Busan 619-705, Korea. Tel. :+82-51-720-2453, Fax :+82-51-720-2456, E-mail : heejkong@korea.kr

This is an Open Access article distributed under the terms of the Creative Commons Attribution Non-Commercial License (http:// creativecommons.org/licenses/by-nc/3.0) which permits unrestricted non-commercial use, distribution, and reproduction in any medium, provided the original work is properly cited.
} 
site recognition (Fortes et al., 1999). The LUC7L is serinearginine-rich protein (SR protein) that localizes in the nucleus through its arginine-serine-rich domain (RS domain) at the C-terminus (Kimura et al., 2004). LUC7L homologs were identified in other species including human Homo sapiens (GenBank accession no. NP_958815.1), red junglefowl Gallus gallus (XP_414651.3), painted turtle Chrysemys picta bellii (XP_005294482) and zebrafish Danio rerio (XP_005162951). Human LUC7L, also called $\mathrm{SR}+89$ or putative SR protein LUC7B1, is closely related to cisplatin resistance-associated over-expressed protein (CROP), which makes anticancer therapy failed (Nishii et al., 2000). Mouse LUC7L plays an important role in the regulation of muscle differentiation (Kimura et al., 2004). LUC7L expression is negatively regulated during the course of development of limb skeletal muscle and during in vitro differentiation of the mouse myoblast cell lines (Kimura et al., 2004). However, the identification and characterization of LUC7L remains to be elucidated in many species.

Korean rose bitterling (Rhodeus uyekii) belongs to the Acheilognathinae subfamily of the Cyprinidae family. It is a common freshwater fish endemic to Korea found in rivers that empty into the Western and Southern Sea of Korea (Kong et al., 2012). This species has been proposed as a candidate for developing ornamental fish because of its small size and beautiful body color (Kang et al., 2005). Genetic studies on the Korean rose bitterling reported the complete mitochondrial genome sequence of $R$. uyekii (Kim et al., 2006) and development of microsatellite makers for evaluation of population genetic diversity (Kim et al., 2014). The $R$. uyekii $\beta$-actin gene has been suggested as a promoter capable of driving constitutive transgene expression (Kong et al., 2014). In this study, we report the identification and molecular characterization of the Luc71 cDNA of Korean rose bitterling (RuLuc71). We analyzed multiple alignments of the deduced RuLUC7L polypeptide sequence and other LUC7L homologs. We investigated the expression of RuLuc71 transcript during early development of Korean rose bitterling and in several tissues of Korean rose bitterling. This study is the first report of molecular and functional analyses of the Korean rose bitterling Luc71 gene.

\section{MATERIALS AND METHODS}

\section{Cloning of Ru-Luc7l from the Rhodeus uyekii}

The RuLuc71 cDNA sequence was isolated from the EST analysis of the Korean rose bittering R. uyekii cDNA library (data not shown). EST clones were isolated from the $R$. uyekii cDNA library using a Plasmid Miniprep Kit (Qiagen), and sequenced using T3 reverse primers (Promega) and an ABI3730xl automatic sequencer (Applied Biosystems). Based on partial sequence sequenced, EST clones were sequenced using designated internal primers (RuLuc71seq 1, 5'-CCT ACT TGG GCC TCC ATG ATA-3'; RuLuc71-seq 2, 5'-ACA GAG AGG CGG GAG AGA TC$3 ')$. The nucleotide sequence was analyzed and compared using the BLASTX search program (http://www.ncbilnlm. nih.gov/BLAST/) .

\section{Multiple sequence alignment and phylogenetic} analysis

The relevant sequences were compared using the BLASTX search program (http://www.ncbi.nlm.nih.gov/BLAST/) and retrieved from GenBank for multiple sequence alignments using CLUSTALW (http://www.genome.jp/tools-bin/clustalw). MEGA (ver. 4) was used to assess similarities among the aligned sequences. A phylogenetic tree based on the deduced amino acid sequences was constructed using a neighbor-joining algorithm, and the reliability of the branching was tested using bootstrap resampling with 1,000 pseudo-replicates.

\section{Quantitative real-time PCR}


Total RNA was prepared from tissues using TRIzol reagent (Invitrogen, Carlsbad, CA, USA) according to the manufacturer's instructions, treated with DNase I (New England BioLabs, Beverly, MA, USA) and quantitatively determined; $500 \mathrm{ng}$ samples were used for reverse transctiption (RT). First-strand cDNA was synthesized using Transcriptor First Strand cDNA Synthesis Kit (Roche). Quantitative real-time PCR was performed using Fast SYBR Green Master Mix (Applied Biosystems, Inc.) and the following forward and reverse primers : RuLuc71, RuLuc71-RT-F (5'-TGG GCC TCC ATG ATA ACG A-3') and RuLuc71-RT-R (5'-GAA GCC CAA GTG CAG TTT GC-3'); and Ruß-actin (GenBank accession no. JQ279058), RubAct-RT-F (5'-GAT TCG CTG GAG ATG ATG CT-3') and RubAct-RT-R (5'-ATA CCG TGC TCA ATG GGG TA-3'). Following an initial 10-min Taq activation step at $95^{\circ} \mathrm{C}$, real-time PCR was performed using the following cycling conditions: 40 cycles of $95^{\circ} \mathrm{C}$ for $10 \mathrm{~s}, 60^{\circ} \mathrm{C}$ for $15 \mathrm{~s}$, and fluorescence reading in an SDS 7500 system (Applied Biosystems, Inc.). Transcript levels were quantified as expression relative to the $\beta$-actin transcript level.

\section{Animals and preparation of tissue}

R. uyekii were collected from the Yangchun River, Uiryung-gun, Gyungnam, Republic of Korea. The fish were maintained at the National Fisheries Research and Development Institute (NFRDI) in Busan, Republic of Korea. The adults were maintained in $40 \mathrm{~L}$ glass aquaria at a density of approximately 20 fish per aquarium. The water was renewed weekly and the temperature in the rearing tanks was maintained at $20 \pm 1^{\circ} \mathrm{C}$. The room was maintained on a 12:12-h light:dark cycle. Adults were fed TetraBits (Tetra) and frozen bloodworms (Advanced Hatchery Technology) twice a day. For RNA extraction, tissues were removed from three $R$. uyekii (mean body weight: $0.75 \pm 0.29 \mathrm{~g}$; mean total length: $4.0 \pm 0.23 \mathrm{~cm}$ ), immediately frozen in liquid nitrogen, and kept separately and stored at $-80^{\circ} \mathrm{C}$ before use.

\section{Statistical analysis}

All data are expressed as means \pm SD $(n=4)$. All data were subjected to a one-way analysis of variance followed by the Holm-Sidak method for multiple ranges testing to determine significant differences among the treatments by SAS 9.1 at the level of $P<0.05$.

\section{RESULTS}

1. Analysis of the nucleotide and deduced amino acid sequences of RuLuc7I

The RuLuc71 cDNA sequence was identified from the expressed sequence tag (EST) analysis of the Korean rose bittering $R$. uyekii cDNA library. The EST clone, RU-24a_D22, of 1688 bp contains 1095-nt ORF encoding a 364-aa protein, preceded by a 120-nt 5' UTR, and followed by a 473-nt 3' UTR with a poly(A) tail (Fig. 1). A search using the BLASTP program (http://www.ncbi.nlm.nih.gov/ blast/Blast.cgi) revealed that the deduced amino acids of RuLuc7l have a serine/arginine-rich region (amino acids $\mathrm{R}^{243}-\mathrm{R}^{355}$ ), which is found commonly in other Luc71 proteins.

\section{Comparision of RuLuc7I with other Luc7I homo-} logs

The deduced amino acids of RuLuc71 were aligned with LUC7L proteins from other species including human using GENETYX software. Pairwise alignment revealed RuLUC7L showed high amino acid identities of $71 \%$ - 97\% with those of other species, including fish, birds, mammals, amphibians and reptiles. Multiple alignments revealed that RuLUC7L shared the high homology with other LUC7L proteins in the N-terminal region, but relatively low in the C-terminal region despite of the presence of serine/argininerich region (Fig. 2). 


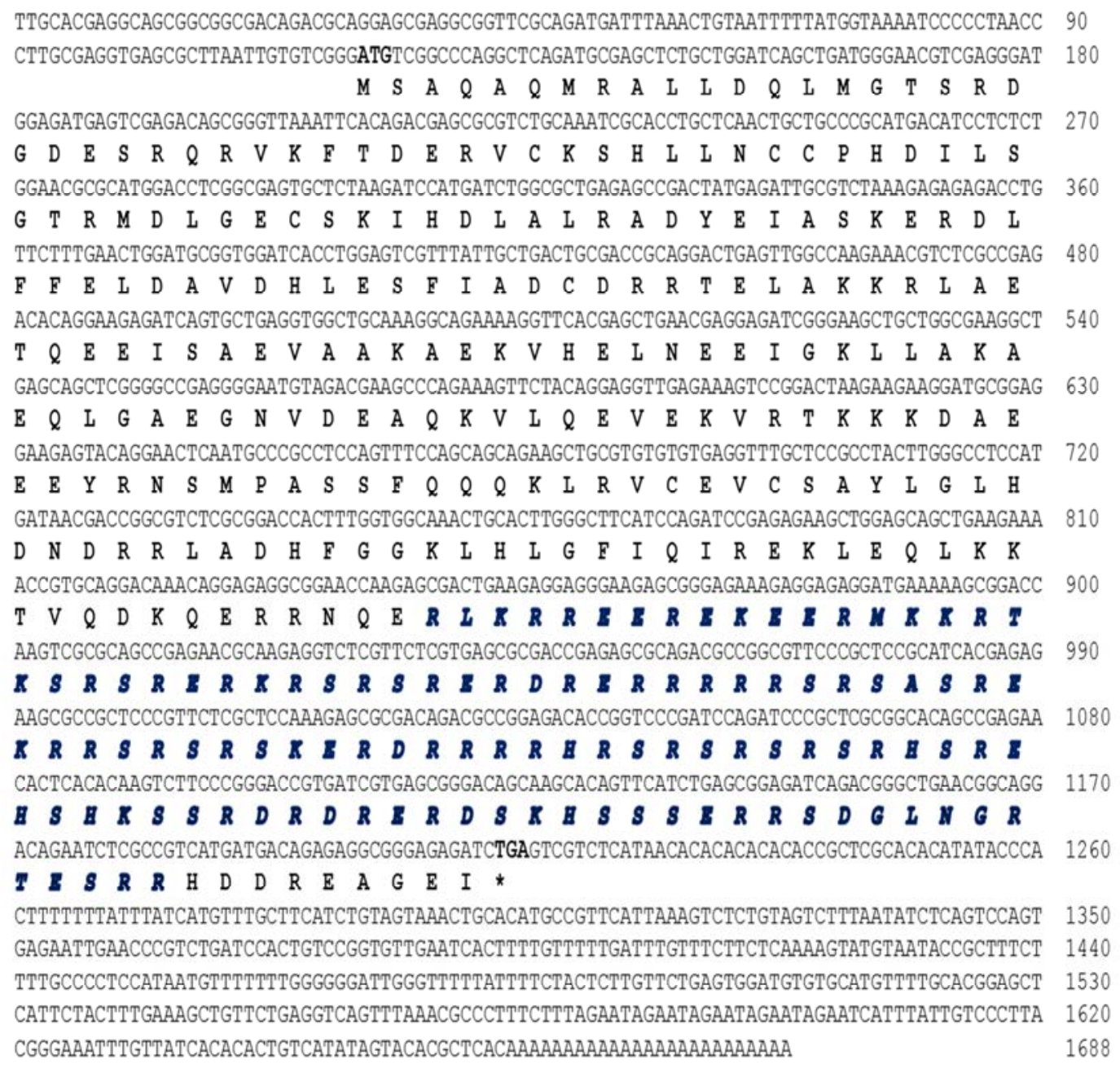

Fig. 1. Nucleotide and deduced amino acid sequences of the Rhodeus uyekii Luc7l. Start and stop codons are shown in bold. The serine/arginine-rich region is in bold and italic.

3. Phylogenetic analysis of RuLuc7l with Luc7l homologs

A phylogenetic analysis was performed based on the deduced amino acid sequence of RuLuc71 and related sequences. The tree indicated clear clustering of LUC7L sequences into two groups: those from Korean rose bitterling, zebrafish, guppy, african bush elephant, green anole, bonobo, killer whale, ground tit, red jungle fowl, painted turtle, western clawed frog, african clawed frog and human. RuLUC7L formed a cluster with LUC7Ls from zebrafish and guppy, and was phylogenetically separate from other species (Fig. 3).
4. Tissue distribution of RuLuc7I mRNA in Korean rose bitterling

Quantitiative real-time PCR was performed to examine the tissue distribution of RuLuc71 mRNA. The expression levels of RuLuc71 mRNA were quantified after normalization to $\beta$-actin as an internal reference gene. Expression of RuLuc71 mRNA was detected in all tissue examined; highly in the brain, stomach and kidney and moderately in the intestine and testis. Levels of the RuLuc71 mRNA in the brain, stomach and kidney were 31.8, 20.0 and 19.8 folds that in muscle where expression was the lowest, respectively (Fig. 4). 


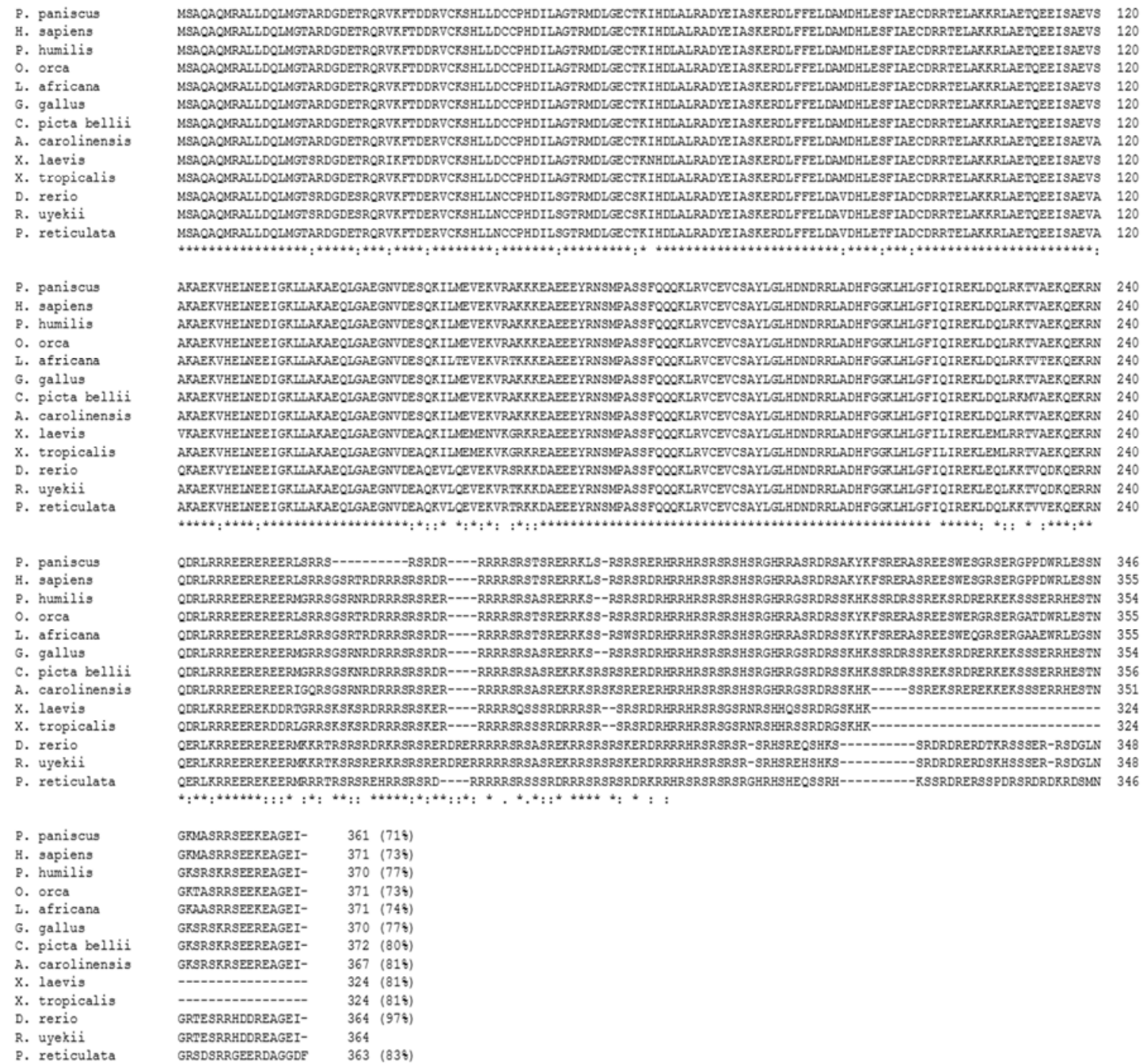

Fig. 2. Multiple alignment of the deduced amino acid sequences of the Rhoedeus uyekii Luc7l with other species. Identical residues in all sequences are indicated in black. The sequences were extracted from GenBank: Danio rerio (NP_001002116.1), Homo sapiens (NP_958815.1), Poecilia reticulata (XP_008434514.1), Loxodonta africana (XP_003417850.1), Anolis carolinensis (XP_003227761.1), Pan paniscus (XP_008959853.1), Orcinus orca (XP_004270427.1), Pseudopodoces humilis (XP_005522898.1), Gallus gallus (XP_414951.3), Chrysemys picta bellii (XP_005294472.1), Xenopus (Silurana) tropicalis (NP_001005458.1), and Xenopus laevis (NP_001090408.1).

\section{Expression analysis of the RuLuc7I mRNA during} early development

The expression of RuLuc71 mRNA during early development of Korean rose bitterling was determined by quantitative real-time PCR at 1, 3, 6, 15 and 21 days postfertilization (dpf). The expression of RuLuc71 mRNA was detected from $1 \mathrm{dpf}$ and moderately increased until $21 \mathrm{dpf}$ during the early development. Levels of the RuLuc71 mRNA at $21 \mathrm{dpf}$ was 2.61 folds that at $1 \mathrm{dpf}$ (Fig. 5).

\section{DISCUSSION}

We previously constructed a cDNA library of the Korean rose bitterling $R$. uyekii and carried out EST analysis of 


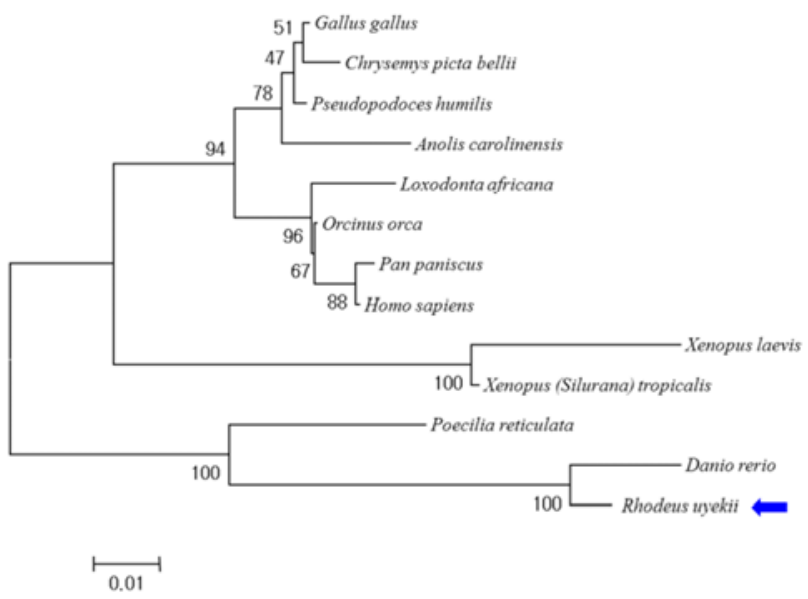

Fig. 3. Phylogenetic analysis of the Korean rose bitterling RuLUC7L and related sequences. GenBank accession numbers for the analyzed sequences are the following: zebrafish (Danio rerio; GenBank accession no. NP_001002116.1), human (Homo sapiens; NP_958815.1), guppy (Poecilia reticulata; XP_008434514.1), African bush elephant (Loxodonta africana; XP_003417850.1), green anole (Anolis carolinensis; XP_003227761.1), bonobo (Pan paniscus; XP_008959853.1), killer whale (Orcinus orca; XP_ 004270427.1), ground tit (Pseudopodoces humilis; XP_005522898.1), red jungle fowl (Gallus gallus; XP_414951.3), painted turtle (Chrysemys picta bellii; XP_005294472.1), western clawed frog (Xenopus (Silurana) tropicalis; NP_001005458.1) and african clawed frog (Xenopus laevis; NP_001090408.1).

approximately 1,000 clones. One of the EST clones contained an open reading frame encoding 364 amino acids which is showing $71-97 \%$ sequence homology with Luc 71 s of other species; zebrafish, guppy, african bush elephant, green anole, bonobo, killer whale, ground tit, red jungle fowl, painted turtle, western clawed frog, african clawed frog and human. The deduced amino acids of RuLuc71 had the serine/arginine-rich region conserved with those of other species. LUC7L belongs to serine/arginine (SR) proteins, characterized by a C-terminal RS domain (Kimura et al., 2004). SR proteins are a conserved protein families involved in RNA splicing, which are commonly found in

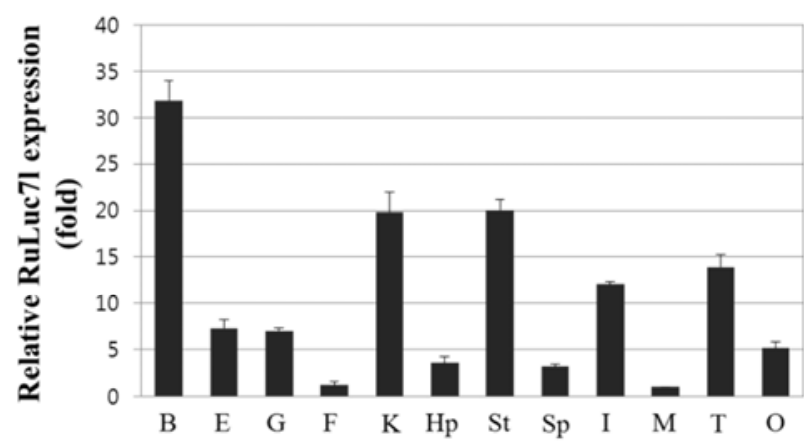

Fig. 4. Tissue distribution of RuLuc7l mRNA. Quantitative real-time PCR analysis was performed with equal amounts of total RNA from tissues of Korean rose bitterling. To determine tissue-specific expression levels, the expression level in each tissue was compared to that in the muscle which was given an arbitrary value of 1 . B, brain; E, eye; G, gill; F, fin; $\mathrm{K}$, kidney; Hp, hepatopancreas; St, stomach; Sp, spleen; I, intestine; M, muscle; T, testis; O, ovary.

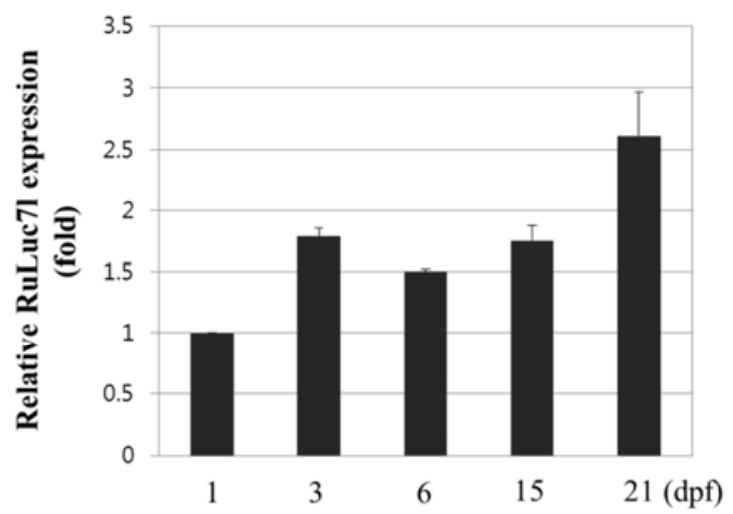

Fig. 5. Expression analysis of the RuLuc7l mRNA during early development. Quantitative real-time PCR analysis was performed with equal amounts of total RNA at $1,3,6,15$, and 21 days post-fertilization (dpf). The Ct values of RuLuc71 used as absolute value. Expression levels were calculated relative to the level of RuLuc7l mRNA at $1 \mathrm{dpf}$.

the nucleus (Zahler et al., 1992). A gene trap mutant protein partially missing its $\mathrm{RS}$ domain, Luc71 ${ }^{\mathrm{GT}}$, lost speckled distribution in the nucleus, suggesting the RS domain is also important for the intranuclear distribution of LUC7L (Kimura et al., 2004). The presence of RS 
Fish Myogenic Regulatory Protein LUC7L: Characterization and Expression Analysis in Korean Rose Bitterling (Rhodeus uyekii)

domain in RuLUC7L suggests that RuLUC7L possibly localizes in the nucleus and might play a role as a regulatory factor in RNA splicing in Korean rose bitterling.

Transcript of RuLuc71 was ubiquitously expressed in all tissue examined and highly expressed in the brain, stomach and kidney. During the early development of Korean rose bitterling, the expression of RuLuc71 was detected after fertilization and increased until $21 \mathrm{dpf}$. This expression pattern is consistent with previous results. Mouse Luc71 was detected in the brain, liver, testis, spleen, and even in the embryo, but not in the skeletal muscle (Kimura et al., 2004). Because LUC7L as an RNA splicing factor is involved in gene expression and regulation (O'Reilly et al., 2013), RuLuc71 might be suggested to be increased during early development. Recent study demonstrated that forced expression of LUC7L protein regulated myogenesis in vitro (Kimura et al., 2004). The level of RuLuc71 mRNA in the muscle was low compared to those of other tissues. These results suggest that RuLUC7L may play a role in the development in a variety of tissue like myogenic regulation.

Cisplatin resistance-associated overexpressed protein (CROP) is the human homologue of yeast LUC7P (Umehara et al., 2003). CROP is a novel putative SR protein sharing the common function with LUC7L. CROP/hLuc7A has been studied as a key molecule of cisplatin resistance of anticancer therapy targeting a variety of malignant tumors because it is overexpressed in cisplatin-resistant cell lines (Umehara et al., 2003). In this context, LUC7L is considered as a possible target for drug resistance in tumor in a manner similar to its mammalian counterparts.

In the present study, we have done the molecular characterization and functional analysis of Luc7l of Korean rose bitterling $R$. uyekii. Sequence and homology analyses showed that the deduced amino acid sequence of RuLuc71 has been evolutionally conserved. Also, its tissue distribution and expression level during early development provide clues for the function involved in gene regulation and development. For understanding biological activity of LUC7L, further studies are required to elucidate the developmental functions of RuLUC7L in myogenesis in $R$. uyekii.

\section{ACKNOWLEDGEMENTS}

This work was supported by a grant from the National Fisheries Research and Development Institute (NFRDI), Republic of Korea.

\section{REFERENCES}

Arnold HH, Braun T (1996) Targeted inactivation of myogenic factor genes reveals their role during mouse myogenesis: a review. Int J Dev Biol 40:345-353.

Fortes P, Bilbao-Cortes D, Fornerod M, Rigaut G, Raymond W, Seraphin B, Mattaj IW (1999) Luc7p, a novel yeast U1 snRNP protein with a role in $5^{\prime}$ splice site recognition. Genes Dev 13:2425-2438.

Hasty P, Bradley A, Morris JH, Edmondson DG, Venuti JM, Olson EN, Klein WH (1993) Muscle deficiency and neonatal death in mice with a targeted mutation in the myogenin gene. Nature 364:501-506.

Johnston I, Cole N, Abercromby M, Vieira V (1998) Embryonic temperature modulates muscle growth characteristics in larval and juvenile herring. J Exp Biol 201:623-646.

Kang EJ, Kim EM, Kim YJ, Lim SG, Sim DS, Kim YH, Park IS (2005) Effect of lidocaine hydrochloride and clove oil as an anaes-thetic on Korean rose bitterling Rhodeus uyekii and oily bittering Acheilognathus koreensis. J Aquac 18:272-279.

Kassar-Duchossoy L, Gayraud-Morel B, Gomes D, Rocancourt D, Buckingham M, Shinin V, Tajbakhsh S (2004) Mrf4 determines skeletal muscle identity in Myf5: Myod double-mutant mice. Nature 431:466-471.

Kim BC, Kang TW, Kim MS, Kim CB (2006) The com- 
plete mitogenome of Rhodeus uyekii (Cypriniformes, Cyprinidae). DNA Seq 17:181-186.

Kim WJ, Shin EH, Kong HJ, Kim HS, Kim BS, Nam BH, Kim YO, Kim CH, Jung H, An CM (2014) Characterization of 18 novel microsatellite markers derived from the rose bitterling (Rhodeus uyekii) genomic libraries. Genet Mol Res 13:8147-8152.

Kimura E, Hidaka K, Kida Y, Morisaki H, Shirai M, Araki K, Suzuki M, Yamamura KI, Morisaki T (2004) Serinearginine-rich nuclear protein Luc7l regulates myogenesis in mice. Gene 341:41-47.

Kong HJ, Kim JL, Kim WJ, Kim HS, Yeo SY, Park JY, An CM (2014) Genomic cloning and promoter analysis of the $\beta$-actin gene from Korean rose bitterling (Rhodeus uyekii). Genes Genom 36:861-869.

Kong HJ, Kim WJ, Kim HS, Lee YJ, Kim CH, Nam BH, Kim YO, Kim DG, Lee SJ, Lim SG, Kim BS (2012) Molecular characterization of a tandem-repeat galectin9 (RuGlec9) from Korean rose bitterling (Rhodeus uyekii). Fish Shellfish Immunol 32:939-944.

Megeney LA, Kablar B, Garrett K, Anderson JE, Rudnicki MA (1996) MyoD is required for myogenic stem cell function in adult skeletal muscle. Genes Dev 10:1173-
1183.

Nishii Y, Morishima M, Kakehi Y, Umehara K, Kioka N, Terano Y, Amachi T, Ueda K (2000) CROP/Luc7A, a novel serine/arginine-rich nuclear protein, isolated from cisplatin-resistant cell line. FEBS Lett 465:153-156.

O’Reilly D, Dienstbier M, Cowley SA, Vazquez P, Drozdz M, Taylor S, James WS, Murphy S (2013) Differentially expressed, variant U1 snRNAs regulate gene expression in human cells. Genome Res 23:281-891.

Rescan PY (2001) Regulation and functions of myogenic regulatory factors in lower vertebrates. Comp Biochem Physiol B 130:1-12.

Rossi G, Messina G (2014) Comparative myogenesis in teleosts and mammals. Cell Mol Life Sci 71:3081-3099.

Umehara H, Nishii Y, Morishima M, Kakehi Y, Kioka N, Amachi T, Koizumi J, Hagiwara M, Ueda K (2003) Effect of cisplatin treatment on speckled distribution of a serine/arginine-rich nuclear protein CROP/Luc7A. Biochem Biophys Res Commun 301:324-329.

Zahler AM, Lane WS, Stolk JA, Roth MB (1992) SR proteins: a conserved family of pre-mRNA splicing factors. Genes Dev 6:837-847. 